\title{
Numerical Investigation on Electron and Ion Transmission of GEM-based Detectors
}

\author{
Purba Bhattacharya ${ }^{1, a}$, Sumanya Sekhar Sahoo ${ }^{1}$, Saikat Biswas ${ }^{1}$, Bedangadas Mohanty ${ }^{1}$, Nayana Majumdar ${ }^{2}$, and \\ Supratik Mukhopadhyay²
}

${ }^{1}$ School of Physical Science, National Institute of Science Education and Research, Jatni, Khurda, India 752050

${ }^{2}$ Applied Nuclear Physics Division, Saha Institute of Nuclear Physics, 1/AF, Bidhannagar, Kolkata, India 700064

\begin{abstract}
ALICE at the LHC is planning a major upgrade of its detector systems, including the TPC, to cope with an increase of the LHC luminosity after 2018. Different R\&D activities are currently concentrated on the adoption of the Gas Electron Multiplier (GEM) as the gas amplification stage of the ALICE-TPC upgrade version. The major challenge is to have low ion feedback in the drift volume as well as to ensure a collection of good percentage of primary electrons in the signal generation process. In the present work, Garfield simulation framework has been adopted to numerically estimate the electron transparency and ion backflow fraction of GEM-based detectors. In this process, extensive simulations have been carried out to enrich our understanding of the complex physical processes occurring within single, triple and quadruple GEM detectors. A detailed study has been performed to observe the effect of detector geometry, field configuration and magnetic field on the above mentioned characteristics.
\end{abstract}

\section{Introduction}

A Time Projection Chamber (TPC) [1] is an ideal device for three-dimensional tracking, momentum measurement and identification of charged particles. They are used in many on-going experiments, including ALICE [2]. Owing to the enormous particle multiplicity per event, very specific requirements are made on the performance of the detectors in harsh radiation environments. Different R\&D activities are currently concentrated on the adoption of the Gas Electron Multiplier (GEM) [3] as the gas amplification stage of the ALICE-TPC upgrade version. Despite the promise, several issues related to the operation of the GEM have to be resolved before it can be finally considered as an option. For example, to keep distortions due to space-charge at a manageable level, a lower ion feedback in the drift volume is required [4]. Again, for a substantial detector gain, it is important that a large fraction of primary electrons partcipate in the avalanche process and contribute to the signal generation. Thus, a proper optimization of the detector geometry, field configuration and gas mixtures are required to have a high electron transparency and low ion backflow.

In the present work, extensive numerical simulations have been carried out to estimate the effects of detector geometry, electric field configurations and magnetic field on electron transparency and ion backflow fraction. To begin with, single and triple GEM configurations have been studied in detail. A good understanding of the complex physics process in these two devices has allowed us to deal with relative ease the quadruple GEM configurations, which is being considered as a possible option for the ALICE TPC. In this manner, it has been possible for us to achieve a understanding of the likely optimum configurations of the quadruple GEMs.

\section{Simulation Tools}

The Garfield [5] simulation framework has been used in the present work. In this framework neBEM [6] is used to carry out 3D electrostatic field simulation. Besides neBEM, the Garfield framework provides interfaces to Magboltz [7] for computing drift, diffusion, Townsend and attachment coefficients.

Table 1: Design parameters of GEM-based detectors.

\begin{tabular}{|c|c|}
\hline Polymer substrate & $50 \mu \mathrm{m}$ \\
\hline Copper coating thickness & $5 \mu \mathrm{m}$ \\
\hline Hole diameter (copper layer) & $70 \mu \mathrm{m}$ \\
\hline Hole diameter (Polymer substrate) & $50 \mu \mathrm{m}$ \\
\hline Hole to hole pitch & $140 / 280 \mu \mathrm{m}$ \\
\hline Drift Gap & $3 \mathrm{~mm}$ \\
\hline $1^{\text {st }}$ Transfer gap & $2 \mathrm{~mm}$ \\
\hline $2^{\text {nd }}$ Transfer gap & $2 \mathrm{~mm}$ \\
\hline $3^{\text {nd }}$ Transfer gap & $2 \mathrm{~mm}$ \\
\hline Induction gap & $2 \mathrm{~mm}$ \\
\hline
\end{tabular}

\footnotetext{
a e-mail: purba.bhattacharya85@gmail.com
} 


\section{Simulation Models}

The design parameters of GEM-based detectors, considered in the numerical work, are listed in table 1 . The model of a basic GEM cell built using Garfield, is shown in figure 1(a). It represents a GEM foil, having two bi-conical shaped holes placed in a staggered manner along with a readout anode and a drift plane on either sides of the foil. The distance between top surface of the GEM and the drift plane is called the drift gap whereas that between the lower surface and the readout plate is named induction gap.

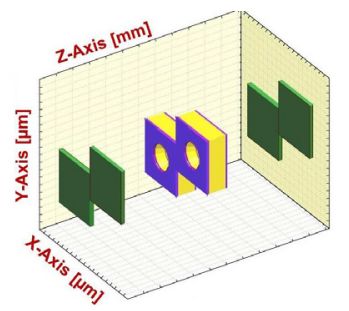

(a)

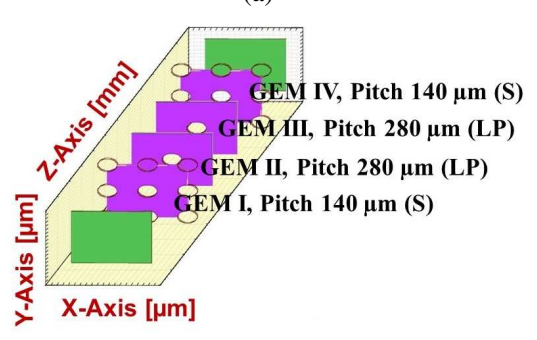

(b)

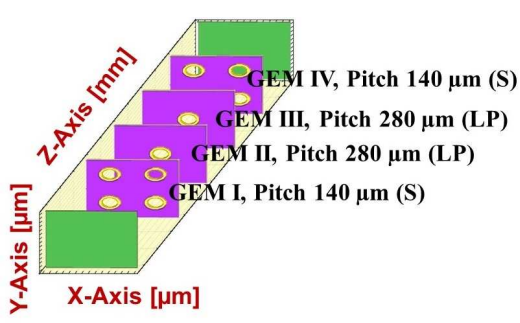

(c)

Figure 1: Simulation model for (a) single GEM and quadruple GEM with (b) aligned holes (denoted as QGemI) (c) misalgned holes (denoted as QGemII)

In comparison to single GEM, in case of multi GEM detector, several GEM foils are placed in between the drift and the read-out plane. The naming scheme used in this work, numbers the foils in the order of the passage of electrons coming from the drift region. The top most GEM is called GEM I and the others are GEM II, GEM III and so on. The gap in between GEM I and II is called transfer gap I and that between GEM II and III is called transfer gap II etc. For example, the simulation models of two different quadruple GEM devices are shown in figure 1. Among the four foils, GEM I and GEM IV, have the pitch of $140 \mu \mathrm{m}$ (denoted as $\mathrm{S}$ ), whereas the middle two foils have a larger pitch of $280 \mu \mathrm{m}$ (denoted as LP). This arrangement, denoted S-LP-LP-S, allows to block ions efficiently by employing asymmetric transfer fields and foils with
Table 2: Voltage configuration of triple, quadruple GEM.

\begin{tabular}{|c|c|c|}
\hline & Triple GEM & Quadruple GEM \\
\hline Drift Field & $0.4 \mathrm{kV} / \mathrm{cm}$ & $0.4 \mathrm{kV} / \mathrm{cm}$ \\
\hline$\Delta V_{G E M I}$ & $250 \mathrm{~V}$ & $275 \mathrm{~V}$ \\
\hline Transfer Field I & $5 \mathrm{kV} / \mathrm{cm}$ & $2 \mathrm{kV} / \mathrm{cm}$ \\
\hline$\Delta V_{\text {GEMII }}$ & $280 \mathrm{~V}$ & $240 \mathrm{~V}$ \\
\hline Transfer Field II & $0.2 \mathrm{kV} / \mathrm{cm}$ & $3 \mathrm{kV} / \mathrm{cm}$ \\
\hline$\Delta V_{\text {GEMIII }}$ & $325 \mathrm{~V}$ & $254 \mathrm{~V}$ \\
\hline Transfer Field III & & $1 \mathrm{kV} / \mathrm{cm}$ \\
\hline$\Delta V_{\text {GEMIV }}$ & & $317 \mathrm{~V}$ \\
\hline Induction Field & $4.5 \mathrm{kV} / \mathrm{cm}$ & $4.5 \mathrm{kV} / \mathrm{cm}$ \\
\hline
\end{tabular}

low optical transparency. An increasing sequence of gas gains down the GEM stack helps reducing the ion backflow since ions created in the inner two layers are blocked more efficiently. Two different geometrical variation in case of four GEM foils have been considered. In the first case (QGEMI), the central hole of the basic unit from all the four GEM foils are perfectly aligned (figure 1(b)). In the other case (QGemII), as shown in figure 1(c), the first and the last foils (S) are aligned with each other whereas the second and third foils (LP) are misaligned with them. In comparison to the quadruple GEM devices, the foils of triple GEM detectors have the same pitch of $140 \mu \mathrm{m}$ and the holes are aligned perfectly. The basic cell structure then has been repeated along both positive and negative $\mathrm{X}$ and Y-Axes to represent a real detector. With the help of these models, the field configuration of the detectors have been simulated using appropriate voltage settings. These are followed by the simulation of electron transmission and ion backflow fraction in $\mathrm{Ne} / \mathrm{CO}_{2} / \mathrm{N}_{2}(90 / 10 / 5)$ gas mixture.

For the calculation of electron transmission, 10,000 electrons have been injected in the drift gap in random positions. These electrons are made to drift towards the GEM foil. The electron transmission has been estimated as the ratio between the number of electrons that reach the anode plate to the number of electrons created in the drift volume.

The electrons during their drift produce avalanche inside the GEM foil. The primary ions in the drift region and the ions created in the avalanche have been considered for the estimation of the backflow fraction, calculated as the ratio between the ions drift back to drift volume to the total number of ions.

\section{Results}

\subsection{Electron Transmission}

Electron transmission can be presented as a function of two mechanisms: electron focusing and transverse diffusion. Electron focusing depends not only on the field ratio, but also on different geometrical parameters. On the other hand, the transverse diffusion is mainly affected by the electric field and the gas composition. Besides that, the 
Table 3: $\epsilon_{\text {coll }}, \epsilon_{e x t}$ and $\epsilon_{t o t}$ of triple, quadruple GEM detectors.

\begin{tabular}{|c|c|c|c|c|c|c|c|c|c|c|}
\hline Geometry & $\mathrm{B}(\mathrm{T})$ & $\epsilon_{\text {collI }}$ & $\epsilon_{\text {extI }}$ & $\epsilon_{\text {collII }}$ & $\epsilon_{\text {extII }}$ & $\epsilon_{\text {collIII }}$ & $\epsilon_{\text {extIII }}$ & $\epsilon_{\text {collIV }}$ & $\epsilon_{\text {extIV }}$ & $\epsilon_{\text {tot }}$ \\
\hline Triple & 0 & 0.996 & 0.329 & 0.324 & 0.042 & 0.989 & 0.402 & & & 0.002 \\
\hline QGemI & 0 & 0.996 & 0.348 & 0.184 & 0.343 & 0.144 & 0.226 & 1.0 & 0.429 & 0.0003 \\
\hline QGemI & 0.5 & 0.996 & 0.349 & 0.175 & 0.361 & 0.2 & 0.326 & 1.0 & 0.357 & 0.0005 \\
\hline
\end{tabular}

electron attachment coefficient, can also influence transmission.

For a single GEM detector, the total electron transmission $\left(\epsilon_{t o t}\right)$ can be identified as the multiplication of two efficiencies, the collection efficiency $\left(\epsilon_{\text {coll }}\right)$ and the extraction efficiency $\left(\epsilon_{\text {ext }}\right)$.

The collection efficiency has been defined as:

$$
\epsilon_{\text {coll }}=\frac{\text { Electrons reached inside the GEM foil }}{\text { Electrons created in drift volume }}
$$

The extraction efficiency has been defined as:

$$
\epsilon_{e x t}=\frac{\text { Electrons reached the readout plane }}{\text { Electrons present inside the GEM foil }}
$$

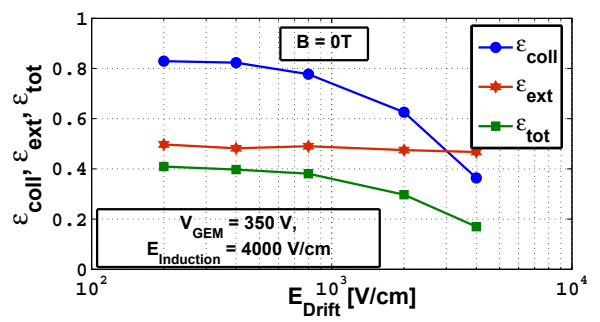

(a)

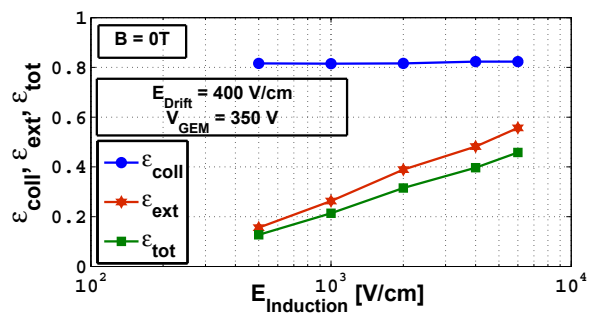

(b)

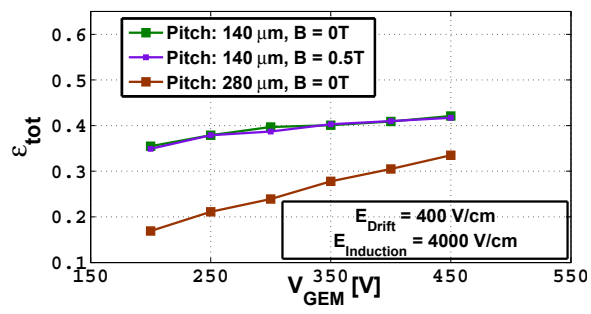

(c)

Figure 2: Variation of $\epsilon_{\text {coll }}, \epsilon_{\text {ext }}, \epsilon_{\text {tot }}$ with (a) $E_{\text {Drift }}$ and (b) $E_{\text {Induction }}$. (c) Variation of $\epsilon_{\text {tot }}$ with $V_{G E M}$. Effect of pitch and magnetic field are shown in (c).

The variations of $\epsilon_{\text {coll }}, \epsilon_{\text {ext }}$ and $\epsilon_{\text {tot }}$ under different field configurations have been plotted in figure 2 . For a fixed $V_{G E M}$ and $E_{\text {Induction }}, \epsilon_{\text {coll }}$ and thus $\epsilon_{\text {tot }}$, decrease with the increase of the drift field, whereas no significant effects of drift field on $\epsilon_{\text {ext }}$ has been observed (figure 2(a)). Similarly, at a fixed $V_{G E M}$ and $E_{\text {Drift }}$, the increase of induction field, increases $\epsilon_{e x t}$ as shown in figure 2(b). The change of $V_{G E M}$ only has effect on $\epsilon_{\text {coll }}$ and thus $\epsilon_{\text {tot }}$ (figure 2(c)). It is also seen from figure 2(c), for the same voltage configuration, the smaller pitch GEM foils is better in terms of higher electron transmission, whereas no significant effect of $0.5 \mathrm{~T}$ magnetic field has been observed.

For the present work, the voltage configuration for triple and quadruple GEM detectors are taken from [4] and [2], respectively and are listed in table 2 . For the multiGEM detectors, the electron transmission can be also identified as the multiplication of collection and extraction efficiencies of the individual GEM foils. $\epsilon_{\text {coll }}$ and $\epsilon_{\text {ext }}$ of individual GEM foils for a triple GEM and a quadruple GEM detectors (QGemI) have been listed in table 3. As is evident for triple GEM detector, the maximum loss happens at GEM II. This is because, the transfer field II is low, which acts as the induction field for the GEM II. Due to this low induction field $\epsilon_{\text {ext }}$ for GEM II is significantly low and thus affect $\epsilon_{t o t}$. For the quadruple GEM detector, for the present voltage configuration, no such low transfer field has been considered. But increase of number of GEM foil worsens the total transmission. $\epsilon_{t o t}$ for the multi-GEM devices is also affected significantly by the variation in geometry. No significant effect of the magnetic field on $\epsilon_{t o t}$ has been observed till now.

\subsection{Ion Backflow Fraction}

As mentioned earlier, the ions created in the avalanche process, drifting back to the drift volume, can disturb the homogeneity of the drift field and, thus, distort the behaviour of the detector. The backflow fraction mainly depends on the field ratio and the transverse spread of the electron avalanche. Thus, a proper optimization of the field in the drift volume, GEM hole and induction regions is necessary to prevent those ions from entering the drift volume.

Table 4: ion collection of triple GEM detectors.

\begin{tabular}{|c|c|c|c|}
\hline GEM I & GEM II & GEM III & Drift \\
\hline 0.0553 & 0.0248 & 0.8368 & 0.0814 \\
\hline
\end{tabular}

The ion backflow of a single GEM can be reduced by decreasing $E_{\text {Drift }}$ because less number of field lines will get out of the hole into the drift volume. At higher $\mathrm{E}_{\text {Drift }}$, the 


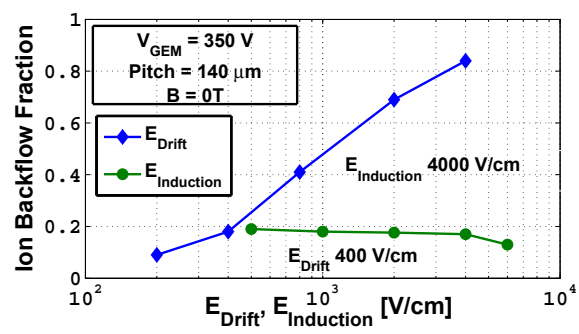

(a)

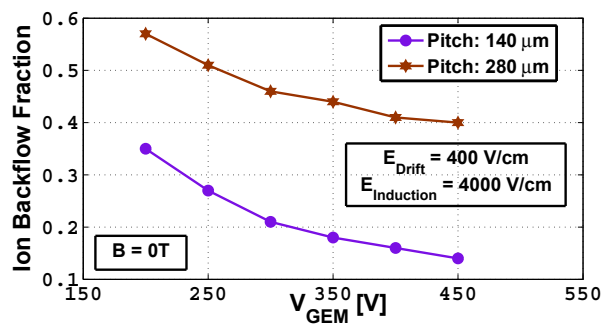

(b)

Figure 3: Variation of ion backflow fraction with (a) $E_{\text {Drift }}$ and $\mathrm{E}_{\text {Induction, (b) }} \mathrm{V}_{\mathrm{GEM}}$. Effect of pitch is shown in (b).

ratio between $\mathrm{E}_{\text {Drift }}$ and $\mathrm{E}_{\mathrm{GEM}}$ is large resulting in the drift of more number of ions into the drift volumm. At higher $\mathrm{E}_{\mathrm{GEM}}$, the ratio between $\mathrm{E}_{\text {Drift }}$ and $\mathrm{E}_{\mathrm{GEM}}$ is small and thus a large fraction of ions is collected at the top surface of the GEM foil. No significant effect of $E_{\text {Induction }}$ has been observed except at the higher $E_{\text {Induction }}$. From figure 3, it is also seen that the GEM foil with smaller pitch is better in terms of lower backflow fraction, whereas no significant effect of 0.5T magnetic field has been observed.

A better suppression of the ion backflow is known to be achieved by using multiple GEM structures. For the triple GEM detector, the ions collection efficiency of three individual GEM foils is listed in table 4. Most of the ions are collected on GEMIII and only $5.4 \%$ ions are able to travel back towards the drift volume. The collection efficiencies of four GEM foils for the two different geometry have been listed in table 5. The backflow for the first case is $\sim 1.7 \%$, whereas the misaligned holes decrease its value to $\sim 0.2 \%$. The effect of magnetic field has been studied in conjuction with the case when there is no magnetic field. No effect of $0.5 \mathrm{~T}$ field on overall backflow fraction has been observed.

\section{Conclusion}

In the upgraded version of the ALICE TPC, the amplification device has been planned to be based on the GEM. Despite its excellent resolution and rate handling capabilities, ion backflow leading to possible space charge accumulation continues to be a source of likely problem for this
TPC. In this work, an attempt has been made to numerically model and optimize the geometrical and electrical configuration of related amplification devices in terms of electron and ion transmission. Study of single GEM detectors shows that higher electron transmission and lower backflow fraction can be obtained with higher GEM voltage, lower drift field and higher induction field. GEM foils with larger pitch gives better electron transmission, as well as less backflow fraction. Multi-GEM devices are found to be better in terms of lower backflow fraction though the electron transmission is affected adversely. Several studies on triple and quadruple GEM detectors with various geometry and field configuration which are likely candidate for the ALICE TPC upgrade, have been performed. No significant effect of $0.5 \mathrm{~T}$ magnetic field has been observed. Further work is necessary to achieve a comprehensive understanding as well as to find out an optimal geometry and field configuration in the scenario of ALICE upgrade. Important details such as space charge and charging up effects, estimates of manufacturing tolerances and defects have been left out of the present computations. In future, we hope to make progress in these areas in order to achieve an even better understanding of these devices.

\section{Acknowledgements}

This work has partly been performed in the framework of the RD51 Collaboration. We wish to acknowledge the members of the RD51 Collaboration for their help and suggestions. We would also like to thank the members of the ALICE-TPC colloboration for their valuable suggestions. We thank our respective Institutions for providing us with the necessary facilities.

Table 5: ion collection of quadruple GEM detectors.

\begin{tabular}{|l|c|c|c|c|c|}
\hline & GEMI & GEMII & GEMIII & GEMIV & Drift \\
\hline $\begin{array}{l}\text { QGemI } \\
\text { OT }\end{array}$ & 0.056 & 0.006 & 0.15 & 0.78 & 0.023 \\
\hline $\begin{array}{l}\text { QGemII } \\
0.0 T\end{array}$ & 0.13 & 0.008 & 0.207 & 0.65 & 0.0021 \\
\hline $\begin{array}{l}\text { QGemII } \\
0.5 T\end{array}$ & 0.128 & 0.008 & 0.201 & 0.66 & 0.0023 \\
\hline
\end{tabular}

\section{References}

[1] V. Lepeltier, Jour. Phy. Conf. Ser. 65012001 (2007).

[2] ALICE Collaboration; TDR 020 (2014).

[3] F. Sauli, Nucl. Instrum. Meth. A 386531 (1997).

[4] M. Ball et al., Jour. Instrum. 9 C04025 (2014).

[5] http://cern.ch/garfield.

[6] http://cern.ch/neBEM.

[7] http://cern.ch/magboltz. 